\title{
BMJ Open One-shot dilation versus serial dilation technique for access in percutaneous nephrolithotomy: a systematic review and meta-analysis
}

\author{
Pan-xin Peng, ${ }^{1,2}$ Shi-cong Lai, ${ }^{3,4}$ Zhen-shan Ding, ${ }^{2}$ Yu-hui He, ${ }^{1,2}$ Li-hua Zhou, ${ }^{1}$ \\ Xu-ming Wang, ${ }^{1,2}$ Guan Zhang ${ }^{1,2,3}$
}

To cite: Peng P, Lai S, Ding Z, et al. One-shot dilation versus serial dilation technique for access in percutaneous nephrolithotomy: a systematic review and meta-analysis. BMJ Open 2019;9:e025871. doi:10.1136/ bmjopen-2018-025871

- Prepublication history and additional material for this paper are available online. To view please visit the journal (http:// dx.doi.org/10.1136/bmjopen2018-025871)

P-P and S-L contributed equally.

P-P and S-L are co-first authors.

Received 4 September 2018 Revised 9 February 2019 Accepted 21 February 2019

Check for updates

(C) Author(s) (or their employer(s)) 2019. Re-use permitted under CC BY-NC. No commercial re-use. See rights and permissions. Published by BMJ.

${ }^{1}$ Peking University China-Japan Friendship School of Clinical Medicine, Beijing, China ${ }^{2}$ Department of Urology, ChinaJapan Friendship Hospital,

Beijing, China

${ }^{3}$ Graduate School of Peking Union Medical College and Chinese Academy of Medical Sciences, Beijing, China ${ }^{4}$ Department of Urology, Beijing Hospital, National Center of Gerontology, Beijing, China

Correspondence to Professor Guan Zhang; gzhang2016@sina.com

\section{ABSTRACT}

Objective The purpose of this study was to systematically review the outcomes of the use of one-shot dilation (OSD) and serial tract dilation for percutaneous nephrolithotomy (PCNL).

Methods A systematic review and meta-analysis was conducted. The randomised controlled trials (RCTs) included in the study were identified from EMBASE, MEDLINE and the Cochrane Central Register of Controlled Trials. The last search was performed on 30 April 2018. Summary effects were calculated as risk ratios (RRs) with $95 \%$ Cls or mean differences (MDs) with $95 \% \mathrm{Cls}$. The endpoints included access time, fluoroscopy time, successful dilation rate, stone-free rate, postoperative decrease in haemoglobin levels, transfusion rate, complication rate and length of postoperative hospital stay. Results A total of seven RCTs were included in the study, with clinical data reported for 697 patients. The overall access time was approximately $110 \mathrm{~s}$ shorter in the OSD group than in the serial dilation group (MD, -110.14 ; $95 \% \mathrm{Cl}-161.99$ to $-58.30 ; p<0.0001)$. The fluoroscopy time was shorter with OSD in all RCTs. In addition, the decrease in postoperative haemoglobin levels was approximately $2.3 \mathrm{~g} / \mathrm{L}$ less in patients in the OSD group than in those in the serial dilation group (MD, -0.23 ; $95 \% \mathrm{Cl}-0.39$ to $-0.07 ; \mathrm{p}=0.004)$. No relationship was found between the successful dilation rate, stone-free rate, transfusion rate, or complication rate and the method of tract dilation.

Conclusion OSD is a safe and efficacious tract dilation technique that can reduce the access time, fluoroscopy time and postoperative decrease in haemoglobin level. No difference was found in the successful dilation rate, stonefree rate, transfusion rate or rate of complications between the OSD and serial dilation groups. The difference in the length of postoperative hospital stay was uncertain. OSD may be a better method of tract creation for PCNL.

\section{INTRODUCTION}

With the development of minimally invasive treatment of urinary calculi, percutaneous nephrolithotomy (PCNL) has become one of the main treatments for large kidney and upper ureteral stones. ${ }^{1}$ One of the most
Strengths and limitations of this study

- This meta-analysis and systematic review was performed via a strict literature search. It was an updated meta-analysis to systematically review the outcomes of one-shot dilation and serial tract dilation for percutaneous nephrolithotomy.

- Seven studies were considered in the final meta-analysis, and several studies with small sample sizes limited the potential analyses.

- Although a systematic search strategy was used, the article language was restricted to English, which may have a resulted in language bias.

Due to a lack of sufficient data, an age-sex adjusted meta-analysis was not conducted.

fundamental steps of PCNL surgery is to establish safe and effective access. However, complications in this process such as tract dilation failure, haemorrhage and perforation of the renal parenchyma or collecting system are not uncommon. ${ }^{2}$ Consequently, it is especially important to identify a simple, effective and safe tract dilation method for clinical application. Currently, the primary dilation methods of access creation in PCNL can be classified as one-shot dilation (OSD) and serial dilation. Although the related systematic reviews were conducted in 2013, only four randomised controlled trials (RCTs) were included in the previous systematic reviews, and the comparison of the two tract dilation methods was not sufficiently comprehensive; therefore, additional RCTs are needed for verification. Currently, the evidence regarding the efficacy and safety of these two methods is still controversial. In recent years, an increasing number of studies have shown that OSD is associated with more advantages than serial dilation, which attracted much attention from urologists. To further compare the safety and efficacy of these two methods, we conducted an 
updated systematic review and meta-analysis of previous RCTs comparing the outcomes of these two tract dilation methods for PCNL.

\section{METHODS}

\section{Patient and public involvement statement}

This study was a systematic review and meta-analysis. Ethics committee approval was not necessary because all data were carefully extracted from existing literature, and this article did not involve handling of individual patient data. In addition, neither patients nor the public were involved in the design and planning of the study.

\section{Literature search}

To assess the clinical efficacy and safety of OSD and the serial dilation technique for PCNL, a comprehensive literature search was performed using EMBASE, MEDLINE and the Cochrane Central Register of Controlled Trials on April 30, 2018. The following MeSH terms and free text words were used: percutaneous nephrolithotomy, PCNL, tract dilation, one-shot, one-step, sequential and serial. These search terms were used alone and in combination. The following search strategy was adopted for each database: ('percutaneous nephrolithotomy' [Mesh] OR 'PCNL') AND ('tract dilatation' [Mesh] OR 'tract dilation' OR 'access creation') AND ('one-shot' [Mesh] OR 'one-step' OR 'single-step' OR 'one-stage' OR 'gradual' OR 'sequential' OR 'serial') (online supplementary file S1). For the study selection, the search strategy was applied based on the Preferred Reporting Items for Systematic Reviews and Meta-analysis statement. ${ }^{3}$ Figure 1 shows the process of identifying RCTs.

\section{Inclusion criteria and study outcomes}

The following inclusion criteria were used: (1) all prospective RCTs compared OSD and serial dilation for PCNL in patients of any age and sex; (2) all patients were in good general condition before surgery and did not have coagulopathy and (3) the article language was restricted to English, and full text or related data could be obtained from the studies. Two authors independently reviewed the titles and abstracts, and differences were discussed with a third author to reach an agreement. The primary outcomes included access time, fluoroscopy time, successful dilation rate and postoperative decreases in haemoglobin levels. The secondary outcomes were transfusion rate, stone-free rate, complication rate and length of postoperative hospital stay.

\section{Data extraction and quality assessment}

Two authors independently extracted the demographic, quality and results data by reading the full-text articles. Data were extracted from the RCTs that met the inclusion criteria. If duplicate research reports were found, the most recent full report was used. Any discrepancies regarding data extraction were resolved by discussion and consultation with senior authors. In addition, we evaluated

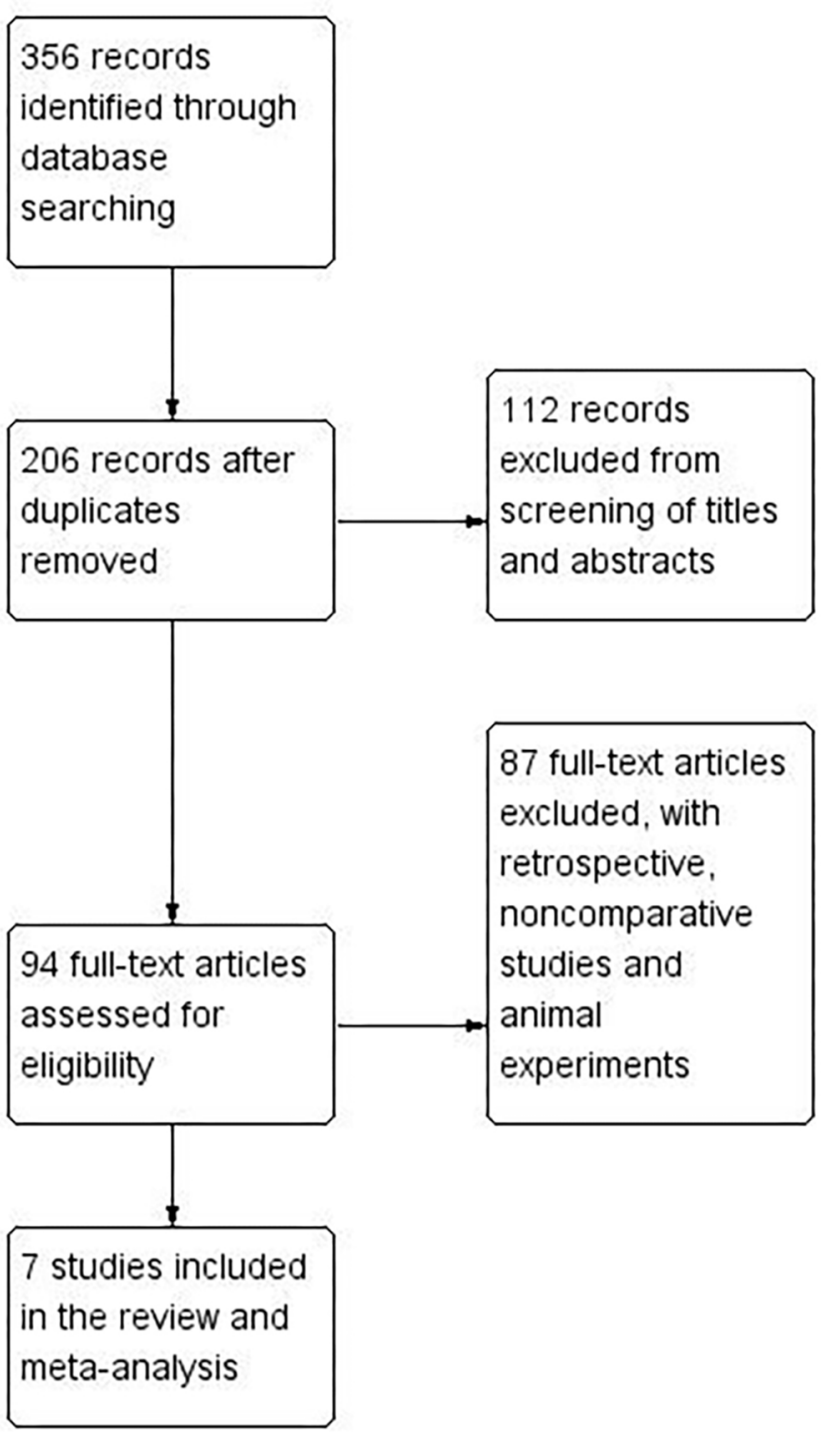

Figure 1 Flowchart of study selection.

the methodological quality of the trials according to the methods recommended by the Cochrane Collaboration. ${ }^{4}$

\section{Data analysis}

We used Review Manager (RevMan V.5.3; Cochrane Collaboration, Copenhagen, Denmark) for the statistical analysis. For dichotomous data, risk ratios (RRs) were used to evaluate the incidence of events, and the results were reported with $95 \%$ CIs. For continuous data, mean differences (MDs) with 95\% CIs were used. Cochrane's $Q$ and the $I^{2}$ statistic were calculated to assess the heterogeneity. When $I^{2}<50 \%$, heterogeneity was considered to be low. When $50 \% \leq I^{2}<75 \%$, heterogeneity was considered moderate. When $I^{2} \geq 75 \%$, heterogeneity was considered high. In cases of low heterogeneity $\left(I^{2}<50 \%\right)$, a fixed-effect meta-analysis was used in conjunction with the study. ${ }^{5}$ In cases of significant heterogeneity $\left(50 \% \leq I^{2}<75 \%\right.$ or $I^{2} \geq 75 \%$ ), a random-effects meta-analysis was used, and studies were individually removed to determine the source of significant heterogeneity. Then, the causes of significant heterogeneity were analysed in detail. Otherwise, 
only a systematic review was performed. A value of $\mathrm{p}<0.05$ was considered statistically significant.

\section{RESULTS}

Based on the search strategies and selection criteria, initial literature searches identified 356 studies across all databases. We eventually included seven RCTs comparing the outcomes of OSD and serial dilation for PCNL in this review. ${ }^{6-12}$ Table 1 shows the basic characteristics of these studies. Several studies were described using only one abstract, but the results were not presented in a usable manner, and the authors declined to provide additional information; therefore, these articles were not included in the results. In all studies, the groups were similar regarding stone location, size and shape, and flexible uretroscopy was not used in any studies. Only a single tract was used in all included studies.

\section{Quality of the included trials}

The overall quality of the included trials was acceptable, although there were some deficiencies in the reporting of methods in some trials. Figure 2 illustrates the risk of bias summary. Random sequence generation was adequate in six trials and unclear in the remaining trial. Allocation concealment was judged to be adequate to minimise selection bias in two trials, unclear in four trials and inadequate in one trial. Blinding of participants and personnel was judged to be adequate to prevent performance bias in two trials, unclear in four trials and inadequate in one trial. Blinding of outcome assessment was judged to be adequate to prevent detection bias in two trials and unclear in five trials. The quality of outcome data reporting was adequate in six trials and unclear in one trial. No selective reporting of outcomes was observed. Other bias was classified as unclear in two and inadequate in five trials.

\section{Access time and fluoroscopy time}

The reported access time varied among the included RCTs. ${ }^{67910}$ The meta-analysis showed that the access time in the OSD group was approximately 110 s shorter than that in the serial dilation group (random-effects analysis: $\mathrm{MD},-110.14 ; 95 \% \mathrm{CI}-161.99$ to -58.30 ; $\mathrm{p}<0.0001$ ) (figure 3A). However, significant heterogeneity was observed $\left(Q=21.86, \mathrm{p}<0.0001, I^{2}=86 \%\right)$.

A sensitivity analysis was performed after Aminisharifi's study ${ }^{9}$ was removed from the analysis. Meta-analysis of this subgroup was supportive of the overall analysis (fixed-effects analysis: MD, $-77.13 ; 95 \% \mathrm{CI}-94.35$ to -59.91 ; $\mathrm{p}<0.00001$ ) (online supplementary figure $\mathrm{S} 1$ ). No significant heterogeneity was found in this subgroup $(Q=1.52$, $\mathrm{p}=0.47, I^{2}=0 \%$ ).

Seven trials reported data regarding fluoroscopy time. ${ }^{6-12}$ All of the trials showed that OSD was associated with significantly decreased fluoroscopy time compared with serial tract dilation. ${ }^{6-12}$ Due to the significant heterogeneity among the studies and the failure to identify

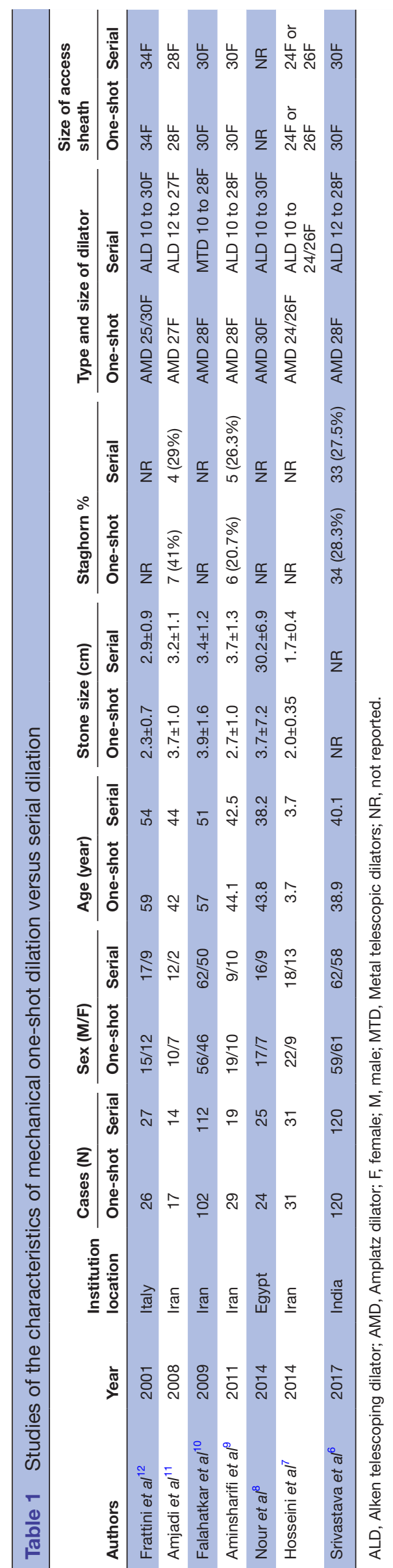




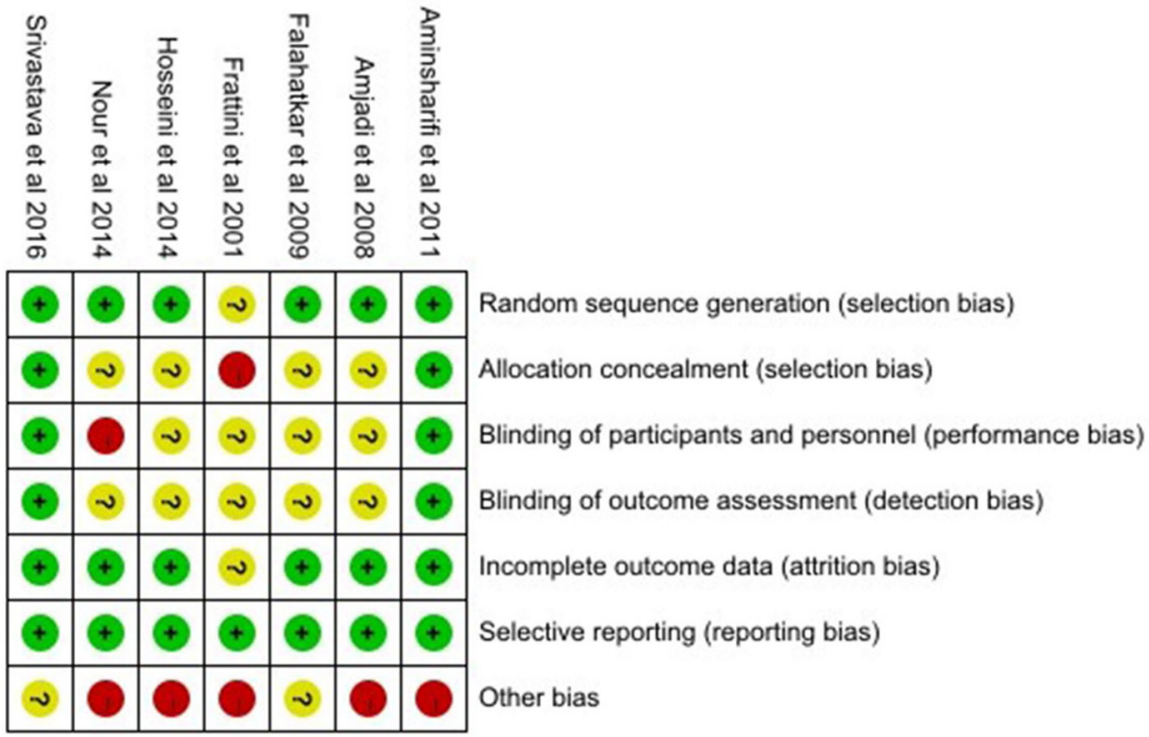

Figure 2 Risk of bias summary: judgements about each risk of bias item for each included study.

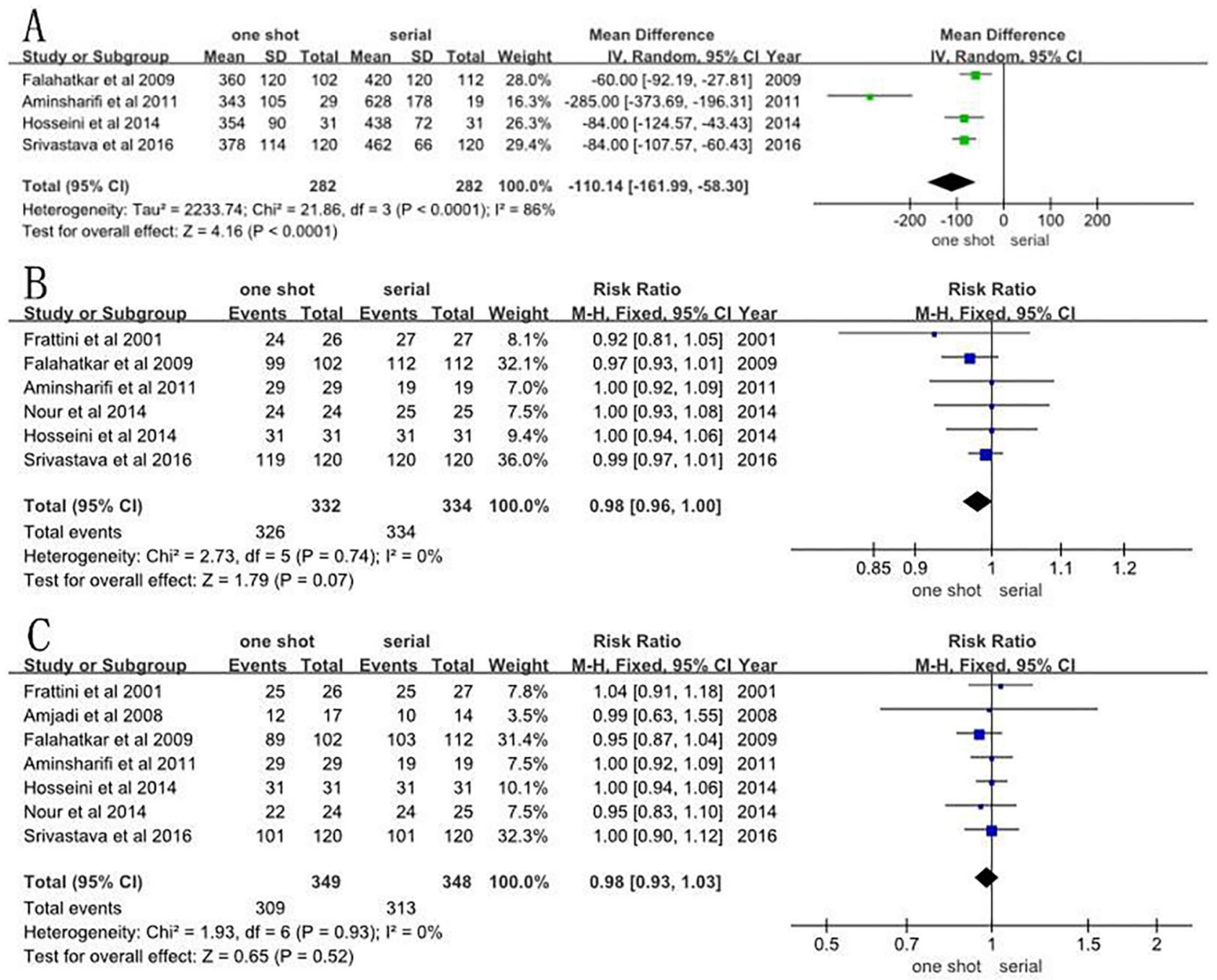

Figure 3 Forest plots illustrating the meta-analysis of outcomes with one-shot tract dilation versus serial tract dilation for percutaneous nephrolithotomy. The outcomes analysed were (A) access time, (B) successful dilation rate and (C) stone-free rate. 
the source of heterogeneity, a meta-analysis was not performed.

\section{Successful dilation rate and stone-free rate}

Six trials reported successful dilation rates. ${ }^{6-11}$ None of the RCTs found significant differences between OSD and serial tract dilatation. The results of the meta-analysis showed that OSD had a slightly lower successful dilation rate than serial tract dilation. However, no significant difference was found (fixed-effects analysis: RR, 0.98; $95 \%$ CI 0.96 to $1.00 ; \mathrm{p}=0.07$ ) (figure $3 \mathrm{~B}$ ). No significant heterogeneity was observed $\left(Q=2.73, \mathrm{p}=0.74, I^{2}=0 \%\right)$.

Seven trials reported stone-free rates, ${ }^{6-12}$ and none found significant differences between OSD and serial tract dilation. The overall stone-free rate was not different between OSD and serial tract dilation (fixed-effects analysis: RR, 0.98; 95\% CI 0.93 to 1.03; $\mathrm{p}=0.52$ ) (figure 3C). Heterogeneity was not observed $\left(Q=1.93, \mathrm{p}=0.93, I^{2}=0 \%\right)$.

\section{Decreases in haemoglobin levels and transfusion rate}

Four RCTs recorded postoperative decreases in haemoglobin levels. ${ }^{691112}$ The haemoglobin levels decreased less in the OSD group than in the serial tract dilation group in two RCTs, but no difference was found in another trial. The results of the meta-analysis showed that OSD significantly reduced haemoglobin decrease compared with serial tract dilation $(\mathrm{MD},-0.23 ; 95 \% \mathrm{CI}-0.39$ to -0.07 ;

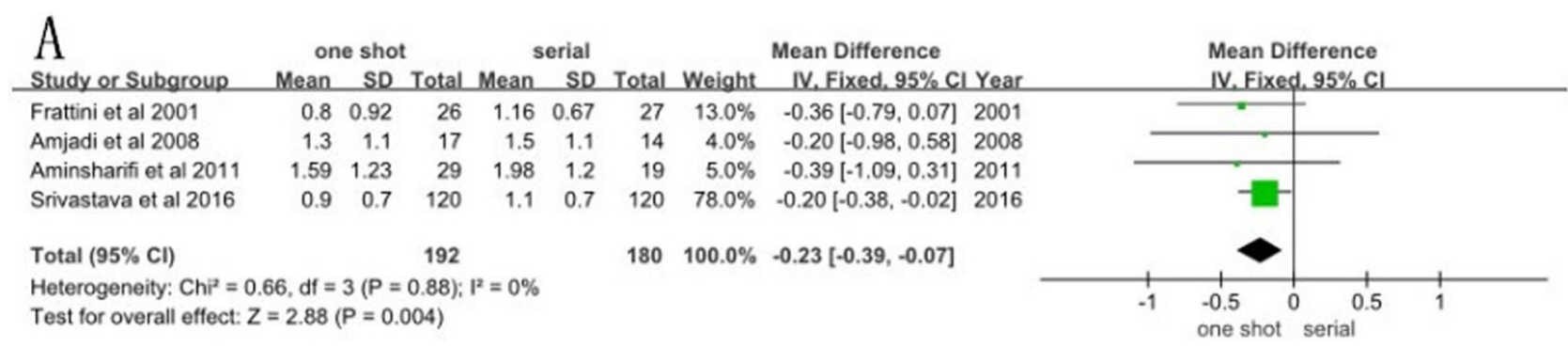

\begin{tabular}{|c|c|c|c|c|c|c|}
\hline B & one sh & & serial & & & Risk Ratio \\
\hline Study or Subgroup & Events & Total & Events & Total & Weight & $\mathrm{M}-\mathrm{H}$, Fixed, $95 \% \mathrm{Cl}$ \\
\hline Frattini et al 2001 & 0 & 26 & 1 & 27 & $11.9 \%$ & $0.35[0.01,8.12]$ \\
\hline Amjadi et al 2008 & 1 & 17 & 1 & 14 & $8.9 \%$ & $0.82[0.06,12.01]$ \\
\hline Falahatkar et al 2009 & 3 & 102 & 5 & 112 & $38.6 \%$ & $0.66[0.16,2.69]$ \\
\hline Srivastava et al 2016 & 4 & 120 & 5 & 120 & $40.5 \%$ & $0.80[0.22,2.91]$ \\
\hline Total $(95 \% \mathrm{Cl})$ & & 265 & & 273 & $100.0 \%$ & $0.69[0.29,1.63]$ \\
\hline Total events & 8 & & 12 & & & \\
\hline \multicolumn{7}{|c|}{ Heterogeneity: $\mathrm{Chi}^{2}=0.25, \mathrm{df}=3(\mathrm{P}=0.97) ; \mathrm{I}^{2}=0 \%$} \\
\hline
\end{tabular}

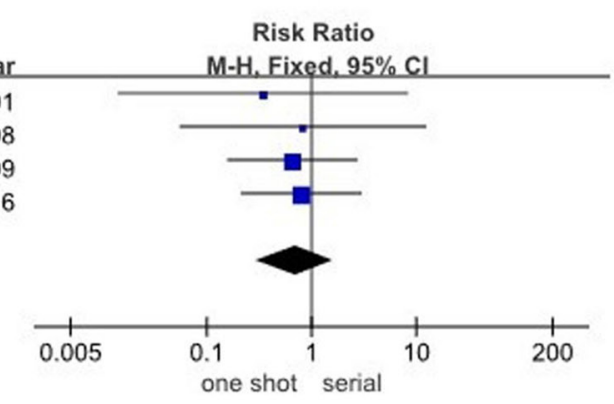

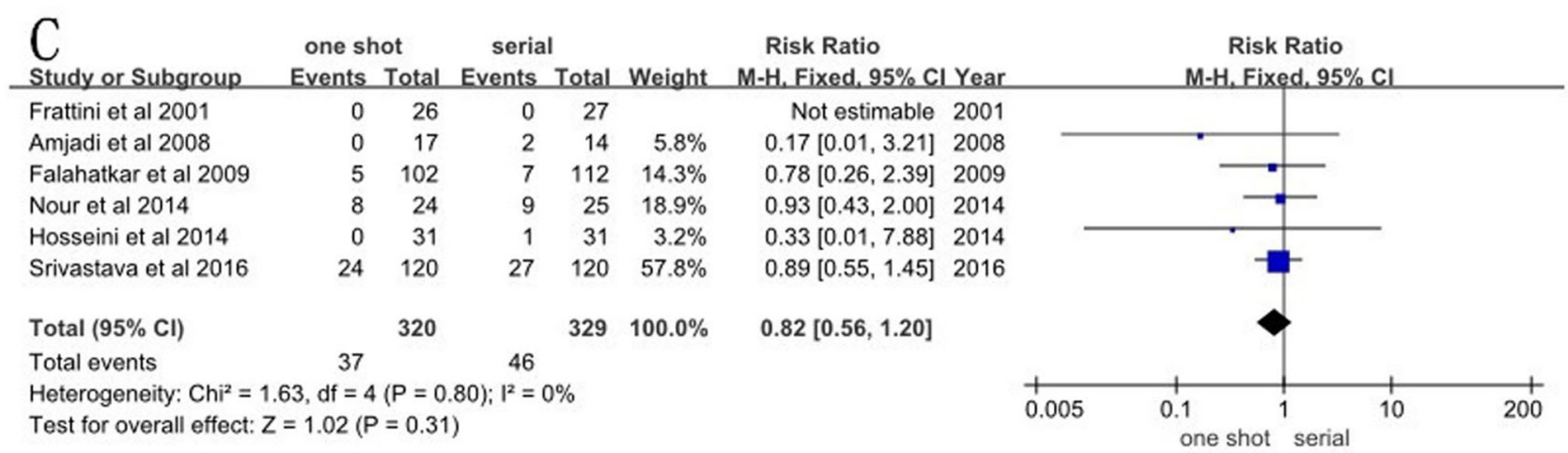

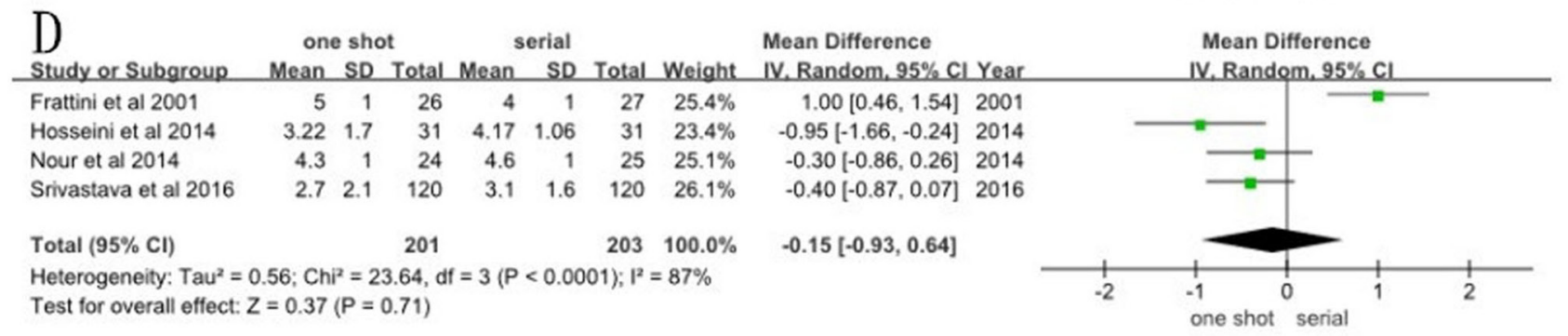

Figure 4 Forest plots illustrating the meta-analysis of outcomes with one-shot tract dilation versus serial tract dilation for percutaneous nephrolithotomy. The outcomes analysed were (A) postoperative decrease in haemoglobin level, (B) transfusion rate, $(C)$ complication rate and (D) length of postoperative hospital stay. 
$\mathrm{p}=0.004) \quad$ (figure $4 \mathrm{~A})$. No heterogeneity was observed $\left(Q=0.66, \mathrm{p}=0.88, I^{2}=0 \%\right)$.

Four trials reported transfusion rates. ${ }^{6}{ }^{10-12}$ Transfusion rates varied among the included studies. No significant difference was found for any trial. Meta-analysis showed that the RR of successful dilation was similar for OSD and serial tract dilation (fixed-effects analysis: RR, 0.69; 95\% CI 0.29 to $1.63 ; \mathrm{p}=0.40$ ) (figure $4 \mathrm{~B}$ ). No significant heterogeneity was observed $\left(Q=0.25, \mathrm{p}=0.97, I^{2}=0 \%\right)$.

\section{Complication rates and length of postoperative hospital stay}

Six RCTs provided complication rates. ${ }^{6-8}{ }^{10-12}$ These RCTs found no relationship between the method of tract dilation and complication rates. A meta-analysis showed that the overall rate of complications was lower in the OSD group than in the serial tract dilation group (fixed-effects analysis: RR, 0.82 ; 95\% CI 0.56 to 1.20 ; $\mathrm{p}=0.31$ ) (figure $4 \mathrm{C})$. Heterogeneity was not observed $(Q=1.63$, $\left.\mathrm{p}=0.80, I^{2}=0 \%\right)$.

Four RCTs provided the length of postoperative hospital stay. ${ }^{6-812}$ One trial reported that serial tract dilation significantly reduced the length of the postoperative hospital stay versus OSD. ${ }^{12}$ Two studies found that OSD was more effective than serial tract dilation in decreasing the length of postoperative hospital stay. ${ }^{78}$ No significant difference was found in one trial. ${ }^{6}$ The meta-analysis showed that the RR of postoperative hospital stay was lower with OSD than with serial tract dilation, but without statistical significance (random-effects analysis: MD, -0.15 ; 95\% CI -0.93 to $0.64 ; \mathrm{p}=0.71$ ) (figure $4 \mathrm{D}$ ). Significant heterogeneity was observed $\left(Q=23.64, \mathrm{p}<0.0001, I^{2}=87 \%\right)$.

A sensitivity analysis was conducted after excluding Frattini's study. ${ }^{12}$ However, the meta-analysis of this subgroup did not support the overall analysis (fixed-effects analysis: $\mathrm{MD},-0.48 ; 95 \%$ CI -0.80 to $-0.16 ; \mathrm{p}=0.003$ ) (online supplementary figure S2). No significant heterogeneity was found in this subgroup $\left(Q=2.21, \mathrm{p}=0.33, I^{2}=0 \%\right)$.

\section{DISCUSSION}

PCNL is the main treatment method for large and complex kidney stones. The creation of a nephrostomy tract is one of the most basic steps of PCNL. This systematic review of seven RCTs including 697 patients examined the evidence for the use of OSD versus serial tract dilation to create access for PCNL. The RCTs showed a statistically significant reduction in access time and fluoroscopy time with OSD but no difference between OSD and serial tract dilatation in terms of the successful dilation rate or transfusion rate. These results are consistent with a previous systematic review performed in 2013. ${ }^{13} 14$ In addition, no difference was observed in the stone-free rate or complication rate. However, postoperative haemoglobin levels decreased less with OSD than with serial tract dilation, which was inconsistent with the results of the previous meta-analysis. ${ }^{14}$ This difference was mainly due to an increased sample size.
Regarding access time, the results of the sensitivity analysis did not change when a study responsible for significant heterogeneity was omitted. ${ }^{9}$ The possible causes of heterogeneity included a small sample size and the surgeon's experience in this study. OSD involving a single dilation of the tract with a 25- or $30 \mathrm{~F}$ dilator is simple and does not require gradual tract dilation. ${ }^{12}$ It saves access time and X-ray exposure during tract creation, thus reducing the operative time and decreasing the risk of radiation damage to patients and operators. In addition, the simple surgical procedures allow surgeons to easily master the technique.

In our experience, successful dilation and stone-free rates are important factors that influence the effectiveness of tract dilation techniques. According to our analysis, the successful dilation rate and stone-free rate of the OSD group were slightly lower than those of the serial group ( $98.2 \%$ vs $100 \%$ and $88.5 \%$ vs $89.9 \%$, respectively); however, our meta-analysis results did not show significant differences regarding the successful dilation rate or the stone-free rate between these two methods. Factors that influence the successful dilation rate may be related to the patient's body mass index, a history of kidney surgery and the surgeon's experience.

The meta-analysis showed that the postoperative haemoglobin levels decreased significantly less in patients who underwent OSD than in those who underwent serial dilation. Kessaris $e t a l^{15}$ found that the amount of intraoperative blood loss caused by the tract dilation technique accounted for half of the total blood loss. The correct puncture path and appropriate tract dilation methods were key decisive factors that determined the amount of intraoperative blood loss. The OSD method may effectively lessen postoperative decreases in haemoglobin levels by reducing the amount of bleeding during surgery. However, the meta-analysis did not find that one particular tract dilation method significantly reduced the transfusion rates of patients, possibly because the sample size of the included studies was not sufficient to detect differences between these two methods. In addition, more high-quality RCTs are required for further study.

In this study, the overall complication rate was $12.8 \%$ in all patients $(11.6 \%$ in the OSD group and $14.0 \%$ in the serial dilation group). The main complications included postoperative urinary tract infections, urine leakage, haemorrhage, haematoma formation and postoperative fever. The difference in complication rates was not statistically significant between the OSD group and the serial dilation group.

The results of the meta-analysis did not show that the OSD technique could significantly reduce the length of the postoperative hospital stay. However, after omitting the study published by Frattini et $a l,{ }^{12}$ we found that the statistical results were significantly changed. The results showed that OSD was more conducive to reducing the length of the postoperative hospital stay than serial dilation. The publication date may be the main source of heterogeneity. In 2001, OSD was a novel method used 
to dilate the nephrostomy access for PCNL. Due to lack of surgical experience, OSD might have caused more parenchymal damage than the serial dilation technique. This damage could prolong the patient's recovery time. With the maturity of the OSD technique, the damage was reduced, thereby shortening the hospital stay. More highquality RCTs are required for further study.

The inclusion criteria of our systematic review specified inclusion of all prospective RCTs comparing the two methods for PCNL for patients of any age or sex; an RCT involving preschool children was also included in the analysis. The results of the study showed that the access and fluoroscopy times in the OSD group were significantly shorter than those in the serial dilation group. These findings are consistent with the results of RCTs involving adults. In addition, the OSD technique significantly shortened the length of the postoperative hospital stay. This study indicated that the OSD method was also safe and effective for preschool children.

It is likely that age and sex caused bias in this study. The age-sex adjusted RR or MD should have been reported in addition to our results. Use of an age-sex adjusted RR or MD in this meta-analysis would have resulted in more appropriate interpretation of our results. However, not all of the seven studies included in our meta-analysis conducted a multivariate analysis. Moreover, the authors of all trials were contacted to obtain the original data to facilitate an in-depth meta-analysis. However, no response was received. Therefore, it is impossible for us to report an age-sex adjusted RR or MD in our manuscript. We hope that further RCTs with detailed data will be available to confirm our conclusions.

Regarding the safety and effectiveness of the OSD technique, some studies have demonstrated that it is equivalent to the serial dilation method for patients with a history of open surgery. ${ }^{11}{ }^{16}$ Furthermore, other important clinical implications of this technique may lie in its cost effectiveness and cost savings. Of note, only one dilator is needed to establish a tract with the OSD technique. The cost of OSD is much lower than that of the serial dilation method, which reduces the economic burden on patients. ${ }^{12}$ Tonshal et al ${ }^{17}$ reported that the cost of the OSD technique is significantly lower than that of the Amplatz sequential dilation technique. Two recent studies have shown that OSD can significantly shorten the length of hospital stay, ${ }^{78}$ which could also reduce the cost of hospitalisation for patients. Reduction in treatment costs can optimise the allocation of medical resources. It is vital to evaluate the cost effectiveness of the two tract dilation techniques in clinical practice, especially for developing countries.

\section{Limitations of this study}

This study had some limitations. First, only seven studies were included in this analysis, and the methodological quality of several studies with small sample sizes was poor or uncertain. These factors might have led to heterogeneity. Second, the experience of the surgeons was not considered, which might have added to the bias. Third, due to the inadequate number of studies included, we did not perform funnel plots for further analysis of publication bias. Despite a systematic search strategy, the article language was restricted to English, which may have resulted in language bias.

\section{CONCLUSION}

The results of this meta-analysis and systematic review suggest that the OSD is a safe and efficacious tract dilation technique that can reduce the access time, fluoroscopy time and postoperative decreases in haemoglobin levels. No differences were observed in the successful dilation rate, stone-free rate, transfusion rate or complication rate between the two techniques. The difference in the length of postoperative hospital stay between the two techniques is uncertain. OSD may be a better method than serial dilation to establish tracts for PCNL. More high-quality RCTs are needed for further study.

Contributors P-xP, S-cL and GZ conceived and designed the experiments. Y-hH and L-hZ extracted the data. P-xP, Z-sD and S-cL analysed the data. Z-sD, Y-hH, $\mathrm{X}-\mathrm{mW}$ and $\mathrm{L}-\mathrm{hZ}$ contributed materials/analysis tools. P-xP and S-cL wrote the paper. GZ critically revised the report.

Funding This study was supported by International S\&T Cooperation Program of China (Grant No 2014DFE30010).

Disclaimer The contents of the present study are solely the responsibility of the author. The funders had no role in study design, data collection and analysis, decision to publish or preparation of the manuscript.

Competing interests None declared.

Patient consent for publication Not required.

Provenance and peer review Not commissioned; externally peer reviewed.

Data sharing statement Data available from the Dryad Digital Repository: https:// doi.org/10.5061/dryad.d5279d1.

Open access This is an open access article distributed in accordance with the Creative Commons Attribution Non Commercial (CC BY-NC 4.0) license, which permits others to distribute, remix, adapt, build upon this work non-commercially, and license their derivative works on different terms, provided the original work is properly cited, appropriate credit is given, any changes made indicated, and the use is non-commercial. See: http://creativecommons.org/licenses/by-nc/4.0/.

\section{REFERENCES}

1. Tomaszewski JJ, Smaldone MC, Schuster T, et al. Factors affecting blood loss during percutaneous nephrolithotomy using balloon dilation in a large contemporary series. J Endourol 2010;24:207-11.

2. Ozok HU, Sagnak L, Senturk AB, et al. A comparison of metal telescopic dilators and Amplatz dilators for nephrostomy tract dilation in percutaneous nephrolithotomy. J Endourol 2012;26:630-4.

3. Liberati A, Altman DG, Tetzlaff J, et al. The PRISMA statement for reporting systematic reviews and meta-analyses of studies that evaluate health care interventions: explanation and elaboration. PLoS Med 2009;6:e1000100.

4. Higgins JP GS. Cochrane handbook for systematic reviews of intervention, vol. 5.1.0 [updated March 2011]. The Cochrane Collaboration, 2011. http:// www.cochrane.org/training cochranehandbook.

5. Mantel N, Haenszel W. Statistical aspects of the analysis of data from retrospective studies of disease. J Natl Cancer Inst 1959;22:719-48.

6. Srivastava A, Singh S, Dhayal IR, et al. A prospective randomized study comparing the four tract dilation methods of percutaneous nephrolithotomy. World J Urol 2017;35:803-7.

7. Hosseini SR, Mohseni MG, Alizadeh F. One shot tract dilation for percutaneous nephrolithotomy: is it safe and effective in preschool children? Urol Int 2014;92:440-3. 
8. Nour HH, Kamal AM, Zayed AS, et al. Single-step renal dilatation in percutaneous nephrolithotomy: A prospective randomised study. Arab J Urol 2014;12:219-22.

9. Aminsharifi A, Alavi M, Sadeghi G, et al. Renal parenchymal damage after percutaneous nephrolithotomy with one-stage tract dilation technique: a randomized clinical trial. J Endourol 2011;25:927-31.

10. Falahatkar S, Neiroomand $\mathrm{H}$, Akbarpour $\mathrm{M}$, et al. One-shot versus metal telescopic dilation technique for tract creation in percutaneous nephrolithotomy: comparison of safety and efficacy. $J$ Endourol 2009;23:615-8.

11. Amjadi M, Zolfaghari A, Elahian A, et al. Percutaneous nephrolithotomy in patients with previous open nephrolithotomy: one-shot versus telescopic technique for tract dilatation. J Endourol 2008;22:423-6.

12. Frattini A, Barbieri A, Salsi P, et al. One shot: a novel method to dilate the nephrostomy access for percutaneous lithotripsy. $J$ Endourol $2001 ; 15: 919-23$.
13. Dehong C, Liangren L, Huawei L, et al. A comparison among four tract dilation methods of percutaneous nephrolithotomy: a systematic review and meta-analysis. Urolithiasis 2013;41:523-30.

14. Li Y, Yang L, Xu P, et al. One-shot versus gradual dilation technique for tract creation in percutaneous nephrolithotomy: a systematic review and meta-analysis. Urolithiasis 2013;41:443-8.

15. Kessaris DN, Bellman GC, Pardalidis NP, et al. Management of hemorrhage after percutaneous renal surgery. J Urol 1995;153(3 Pt 1):604-8.

16. Ziaee $\mathrm{SA}$, Karami H, Aminsharifi A, et al. One-stage tract dilation for percutaneous nephrolithotomy: is it justified? J Endourol 2007;21:1415-20.

17. Tonshal S, Arun Kumar B, Venkatraman M. MP-10.18 One Shot Versus Amplatz Sequential Fascial Dilatation Technique for Tract Creation in PCNL. Urology 2011;78:S108. 